\section{ABVD plus rituximab versus ABVD alone for advanced stage, high-risk classical Hodgkin lymphoma: a randomized phase 2 study}

Based on several randomized studies comparing doxorubicin, bleomycin, vinblastine, and dacarbazine (ABVD) with other multi-drug regimens, $A B V D$ is the standard of care for newly diagnosed, advanced stage, classical Hodgkin lymphoma (cHL). While the cure rate with ABVD approximates $75 \%$ in patients with low-risk disease, only $55 \%$ of patients with high-risk, advanced $\mathrm{cHL}$, defined as an international prognostic score (IPS) greater than 2 , will be disease-free at 3 years, highlighting the need for more effective therapeutic strategies.

In up to $20-30 \%$ of patients with cHL, Reed-Sternberg (RS) cells express CD20. ${ }^{2}$ CD20 is also expressed on the precursors of RS cells and in B-lymphocytes, exerting a pro-tumoral activity in the tumor microenvironment.

Pre-clinical studies have shown that the anti-CD20 monoclonal antibody rituximab may be an effective therapeutic strategy in cHL, both by direct killing of RS cells and by targeting the surrounding microenvironment., As a single agent rituximab has activity in relapsed $\mathrm{cHL}^{5,6}$ and its combination with ABVD (R-ABVD) as frontline therapy for patients with advanced cHL resulted in a 3year event-free survival of $77 \%$, with a $22 \%$ absolute improvement as compared to historical data with ABVD. ${ }^{7,8}$ We present here the results of a multicenter, open-label, randomized, phase 2 study (NCT00654732), comparing $\mathrm{R}-\mathrm{ABVD}$ to $\mathrm{ABVD}$ as initial treatment of patients with advanced stage, high-risk (IPS >2) cHL.

Eligible patients were required to have histologically confirmed, chemotherapy-naïve, advanced-stage cHL (stage III or IV disease), and an IPS $>2$. The study protocol was approved by the institutional review boards of all institutions involved, and conducted in accordance with the principles of the Declaration of Helsinki. All patients provided written informed consent to participation in the study.

R-ABVD and ABVD were given as previously described. The responses were assessed using the 2007 Revised Response Criteria for Malignant Lymphoma (including positron emission tomography but not Deauville criteria; no central review was required per protocol), and toxicity was graded by Common Terminology Criteria for Adverse Event (CTCAE) version 1 . Based on a risk of febrile neutropenia of $10-20 \%$, in the case of an absolute neutrophil count $<1 \times 10^{9} / \mathrm{L}$, the next cycle of treatment was delayed until an absolute neutrophil count $>1 \times 10^{9} / \mathrm{L}$ was reached, and growth factor was added to the following cycles. A tumor was considered positive if any RS cells expressed CD20 by immunohistochemistry.

Based on the results of the original, single-arm, phase 2 study, the primary endpoint was a $22 \%$ increase in 3 -year event-free survival. Assuming a two-sided type I error rate of 0.05 , a trial with 54 patients in each arm was calculated to have $80 \%$ power to detect such an increase; unfortunately, because of the low accrual rate, the study was closed prematurely and the target population sample was not reached. Categorical variables were compared using a $\chi^{2}$ or Fisher exact test. Event-free survival was defined as the time from entry into the study to disease progression, relapse, or death from any cause. Overall survival was calculated from study entry to death from any cause. Survival curves were calculated according to the method of Kaplan and Meier, and compared using the
Table 1. Patients' baseline characteristics.

Number $(\%)$
Patients $(N=58) \quad$ R-ABVD $(n=26) \quad$ ABVD $(n=32)$

Age, years

\begin{tabular}{lcc}
$<45$ & $15(58)$ & $16(50)$ \\
$\geq 45$ & $11(42)$ & $16(50)$ \\
Male & $14(54)$ & $22(69)$ \\
Female & $12(46)$ & $10(31)$ \\
Stage & & \\
III & $4(15)$ & $9(28)$ \\
IV & $22(85)$ & $23(72)$ \\
International Prognostic Score & \\
3 & $13(50)$ & $13(41)$ \\
$\geq 4$ & $13(50)$ & $19(59)$ \\
\hline RS CD20 & & \\
positive & $5(19)$ & $10(31)$ \\
negative & $21(81)$ & $22(69)$ \\
\hline
\end{tabular}

RS: Reed Sternberg cells.

log-rank test. All $P$-values are two-sided and considered statistically significant if $\leq 0.05$ (IBM SPSS 21 ).

Fifty-eight patients were enrolled between April 2008 and October 2012: 26 were randomized to receive RABVD (19\% of whom had CD20-positive RS cells), and 32 to receive ABVD (31\% of whom had CD20-positive RS cells). The patients' baseline characteristics are shown in Table 1.

The intended six cycles of treatment were completed by $85 \%$ of patients in the R-ABVD arm and $84 \%$ in the ABVD arm $(P=0.64)$. Three patients enrolled in the $\mathrm{R}-$ ABVD arm received radiation after this treatment $(12 \%$ versus $0 \%, P=0.08)$. The overall response rates were $88 \%$ and $94 \%(P=0.40)$ in the two arms, while the complete remission rates were $80 \%$ and $81 \%(P=0.64)$, respectively. Among the patients receiving R-ABVD, no increase in complete remission rate was observed based on whether the patients' RS cells were or were not CD20 positive ( $80 \%$ versus $81 \%$, respectively; $P=0.90)$.

The most common grade 3-4 toxicities (incidence $\geq 5 \%$ ) were neutropenia $(50 \%$ in the R-ABVD arm versus $41 \%$ in the ABVD arm; $P=0.60)$, anemia ( $4 \%$ versus $6 \% ; P=1$ ), thrombocytopenia ( $4 \%$ versus $6 \% ; P=1)$, infections $(15 \%$ versus $3 \% ; P=0.16)$, respiratory complications $(0 \%$ versus $6 \% ; P=0.50)$, and neuropathy ( $4 \%$ versus $9 \% ; P=0.62$ ).

After a median follow-up of 67 months (range, 3-113 months), eight (31\%) patients progressed in the R-ABVD arm (4 of whom died), and six $(19 \%)$ in the ABVD arm (2 of whom died). Death in the absence of disease progression was observed in one patient in the R-ABVD arm (total of 9 events) and four patients in the ABVD arm (10 events), and the 3-year event-free survival rates were $63 \%$ and $75 \%$, respectively $(P=0.60)$ (Figure $1 \mathrm{~A})$. The highest 3-year free survival rate $(80 \%)$ was observed among patients with CD20-positive RS cells treated with R-ABVD, and the lowest $(60 \%)$ among patients with CD20-negative RS cells treated with the same regimen (Figure 1B).

At the most recent follow-up, five patients (19\%) had died in the R-ABVD arm and six (19\%) in the ABVD arm. The 3-year overall survival rate was $85 \%$ in both groups $(P=0.94)$ (Figure 1C). Among patients treated with RABVD, the causes of death were known for three 
A

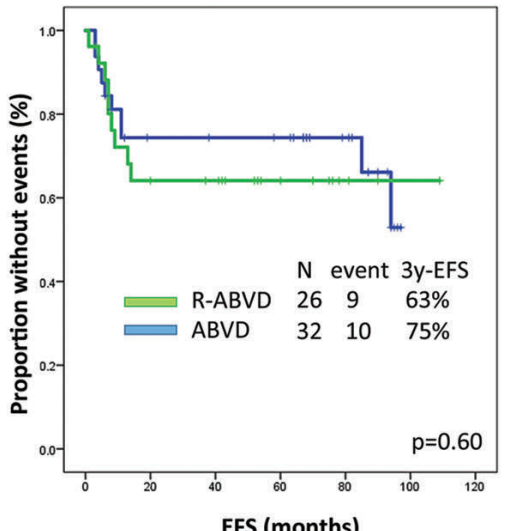

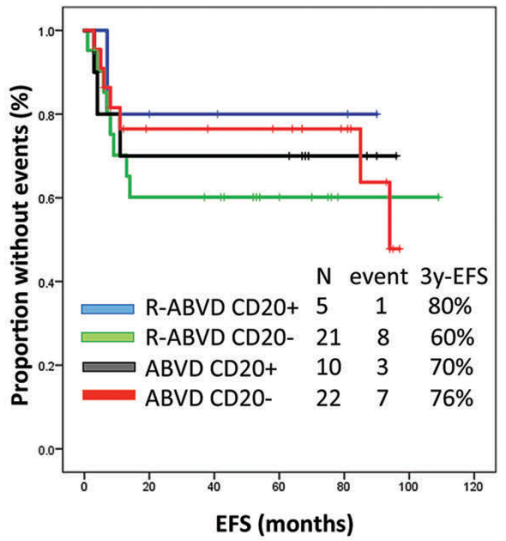

C

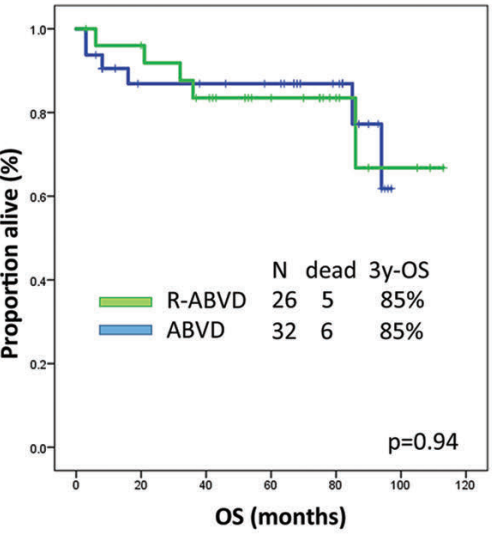

Figure 1. Survival of patients with advanced stage, high-risk classical Hodgkin lymphoma treated with ABVD alone or with ABVD plus rituximab. (A) Event-free survival (EFS) by treatment arm. (B) EFS by treatment arm and CD20 status. (C) Overall survival (OS) by treatment arm. ABVD: doxorubicin, bleomycin, vinblastine, and dacarbazine; R-ABVD: rituximab plus doxorubicin, bleomycin, vinblastine, and dacarbazine.

patients, and were disease progression $(\mathrm{n}=2)$ and respiratory failure $(n=1)$; among patients treated with ABVD, the causes of death were known for five patients, and were disease progression $(n=2)$, respiratory failure $(n=2)$ and metastatic esophageal cancer $(n=1)$.

While we acknowledge that its early termination significantly affected the statistical power and quality of the evidence provided, this is the first randomized study comparing R-ABVD to ABVD as initial treatment of patients with advanced stage, high-risk cHL. Despite the promising results observed in previous single-arm, phase 2 studies, ${ }^{7,8}$ no significant differences in outcomes were observed with the use of R-ABVD as compared to ABVD, and the primary endpoint of the study was not met. Of interest, similar randomized studies, comparing the combination of rituximab with bleomycin, etoposide, doxorubicin, cyclophosphamide, vincristine, procarbazine, and prednisone (BEACOPP) to BEACOPP alone in a similar population also failed to show any improvement in survival. ${ }^{9,10}$

In our study, we observed that patients with CD20positive RS cells treated with R-ABVD had an increased 3 -year event-free survival. While this study was underpowered to detect an increase in event-free survival in CD20-positive cHL, these results suggest that a larger study limited to this population would be useful for ascertaining whether addition of rituximab may improve treatment efficacy in these patients. The malignant cells of the lymphocyte-predominant subtype of Hodgkin lymphoma universally express CD20, and the addition of rituximab to chemotherapy resulted in a 5 -year eventfree survival of $88.5 \%$ in a population with this subtype of lymphoma. ${ }^{11}$ No difference in response rate based on CD20 status was observed in the pilot study of singleagent rituximab for the treatment of patients with relapsed cHL. ${ }^{5}$ However, in the original, single-arm, phase 2 study of frontline R-ABVD, a higher 3-year event-free survival rate $(92 \%$ versus $77 \%$ ) was reported for patients with CD20-positive RS cells. ${ }^{7}$ While there is a pre-clinical rationale for using rituximab in patients with CD20-negative RS cells, based on CD20 expression on precursor RS cells and pro-tumoral B-lymphocytes, ${ }^{4,12}$ further studies focusing only on patients with CD20-positive RS cells may portend better results.
It is important to note that in our study the rate of grade 3-4 neutropenia was higher in the R-ABVD arm than in the ABVD arm, although the difference was not statistically significant. Rituximab can induce immunemediated neutropenia, increasing the risk of neutropenia and febrile neutropenia associated with the use of ABVD. ${ }^{13}$ This may explain the overall shorter event-free survival observed in our study with R-ABVD, as compared to ABVD, despite which the former arm still compared favorably when the analysis was stratified by RS CD20-status.

In addition, RS CD20 status did not affect event-free survival among patients in the ABVD arm, suggesting that its positivity may represent a predictive, rather than a prognostic, factor.

The frontline treatment of patients with advanced stage cHL is quickly moving to targeted therapy. In the phase 3 international ECHELON-1 study, the combination of brentuximab vedotin (BV) with doxorubicin, vinblastine, and dacarbazine (AVD) as frontline treatment in patients with advanced stage cHL met the primary endpoint of increased modified event-free survival. ${ }^{14}$ In addition, cohort D of the Checkmate 205 study included patients with newly diagnosed, advanced stage cHL who, after frontline treatment with four cycles of nivolumab, received six cycles of the combination of nivolumab and AVD: the overall response rate of these patients was $86 \%$ and their complete remission rate was $80 \% .{ }^{15}$ Finally, a phase I trial evaluating the combination of nivolumab and $\mathrm{BV}$ as frontline treatment for elderly patients with cHL not eligible for chemotherapy is underway (NCT02758). Longer follow-up is needed to demonstrate durability of response with these novel treatment options in the frontline setting.

In upcoming chemotherapy-free trials, the investigation of the activity of rituximab in combination with biological agents and/or immunotherapy is warranted, particularly in patients with CD20-positive RS cells.

Paolo Strati, ${ }^{1}$ Michelle A. Fanale, ${ }^{2,3}$ Yasuhiro Oki, ${ }^{2}$ Francesco Turturro, ${ }^{2}$ Luis E Fayad, ${ }^{2}$ Nancy L. Bartlett, ${ }^{4}$ Douglas E. Gladstone,, Yvette L. Kasamon, Carol S. Portlock, Wyndham H. Wilson, ${ }^{7}$ Andre Goy, Anas Younes ${ }^{6}$ and Hun Ju Lee 
'Division of Cancer Medicine, The University of Texas MD Anderson Cancer Center, Houston, TX; ${ }^{2}$ Department of Lymphoma and Myeloma, The University of Texas MD Anderson Cancer Center, Houston, TX; ${ }^{3}$ Seattle Genetics, WA; ${ }^{4}$ Division of Hematology and Oncology, Washington University School of Medicine, St. Louis, MO; ${ }^{5}$ Sidney Kimmel Comprehensive Cancer Center, The Johns Hopkins University School of Medicine, Baltimore, MD; ${ }^{6}$ Division of Hematologic Oncology, Memorial Sloan Kettering Cancer Center, New York, NY; ${ }^{7}$ Lymphoid Malignancies Branch, Center for Cancer Research, National Cancer Institute, Bethesda, MD and ${ }^{8}$ Lymphoma Division, John Theurer Cancer Hackensack University Medical Center, Hackensack, NJ, USA

Correspondence:hunlee@mdanderson.org doi:10.3324/haematol.2018.199844

Information on authorship, contributions, and financial \& other disclosures was provided by the authors and is available with the online version of this article at www. haematologica.org.

\section{References}

1. Loeffler M, Brosteanu O, Hasenclever D, et al. Meta-analysis of chemotherapy versus combined modality treatment trials in Hodgkin's disease. International Database on Hodgkin's Disease Overview Study Group. J Clin Oncol. 1998;16(3):818-829.

2. Rassidakis GZ, Medeiros LJ, Viviani S, et al. CD20 expression in Hodgkin and Reed-Sternberg cells of classical Hodgkin's disease: associations with presenting features and clinical outcome. J Clin Oncol. 2002;20(5):1278-1287.

3. Kim S, Fridlender ZG, Dunn R, et al. B-cell depletion using an antiCD20 antibody augments antitumor immune responses and immunotherapy in nonhematopoetic murine tumor models. J Immunother. 2008;31(5):446-457.

4. Jones RJ, Lin L, Gocke C, et al. Clonotypic B cells circulate in Hodgkin's lymphoma (HL). Blood. 2006;108(11):143a.

5. Younes A, Romaguera J, Hagemeister F, et al. A pilot study of ritux- imab in patients with recurrent, classic Hodgkin disease. Cancer. 2003;98(2):310-314.

6. Rehwald U, Schulz H, Reiser M, et al. Treatment of relapsed CD20+ Hodgkin lymphoma with the monoclonal antibody rituximab is effective and well tolerated: results of a phase 2 trial of the German Hodgkin Lymphoma Study Group. Blood. 2003;101(2):420-424.

7. Younes A, Oki Y, McLaughlin P, et al. Phase 2 study of rituximab plus ABVD in patients with newly diagnosed classical Hodgkin lymphoma. Blood. 2012;119(18):4123-4128.

8. Kasamon YL, Jacene HA, Gocke CD, et al. Phase 2 study of rituximab-ABVD in classical Hodgkin lymphoma. Blood. 2012;119(18):4129-4132.

9. Borchmann P, Haverkamp H, Lohri A, et al. Progression-free survival of early interim PET-positive patients with advanced stage Hodgkin's lymphoma treated with BEACOPPescalated alone or in combination with rituximab (HD18): an open-label, international, randomised phase 3 study by the German Hodgkin Study Group. Lancet Oncol. 2017;18(4):454-463.

10. Gallamini A, Patti C, Viviani S, et al. Early chemotherapy intensification with BEACOPP in advanced-stage Hodgkin lymphoma patients with a interim-PET positive after two ABVD courses. Br J Haematol. 2011;152(5):551-560.

11. Fanale MA, Cheah CY, Rich A, et al. Encouraging activity for R$\mathrm{CHOP}$ in advanced stage nodular lymphocyte-predominant Hodgkin lymphoma. Blood. 2017;130(4):472-477.

12. Younes A, Carbone A. Clinicopathologic and molecular features of Hodgkin's lymphoma. Cancer Biol Ther. 2003;2(5):500-507.

13. Voog E, Morschhauser F, Solal-Celigny P. Neutropenia in patients treated with rituximab. N Engl J Med. 2003;348(26):2691-2694; discussion 2691-2694.

14. Connors JM, Radford JA. Brentuximab vedotin for stage III or IV Hodgkin's lymphoma. N Engl J Med. 2018;378(16):1560-1561.

15. Ramchandren R, Fanale MA, Rueda A, et al. Nivolumab for newly diagnosed advanced-stage classical Hodgkin lymphoma (cHL): results from the phase 2 checkmate 205 study. Blood. 2017;130(Suppl 1):651-651. 